Bangladesh J. Zool. 49 (2): 243-255, 2021

ISSN: 0304-9027

eISSN: $2408-8455$

\title{
A KARYOLOGICAL STUDY OF TENUALOSA ILISHA (HAMILTON, 1822) FROM THE CONFLUENCE OF PADMA AND MEGHNA RIVER OF BANGLADESH
}

\author{
Md. Abu Horayra ${ }^{1}$, Md. Arafat Rahman Khan ${ }^{1}$, Faria Akbar ${ }^{2}$, Susmita Saha ${ }^{2}$, \\ Mehrab Chowdhury ${ }^{1}$ and Kazi Nahida Begum ${ }^{2 *}$ \\ Department of Zoology, Faculty of Life and Earth Sciences, Jagannath University, \\ Dhaka-1100, Bangladesh
}

\begin{abstract}
Tenualosa ilisha (Hamilton, 1822), commonly known as Hilsha shad is a valuable and highly acceptable species in terms of their high flavored properties. Hilsha shad has striking morpho-genetical adaptation to heterogeneous habitats across their migratory routes. Cytogenetic analysis demonstrates the changes in chromosomes. But none was focused on the cytogenetic analysis of $T$. ilisha in Bangladesh. T. ilisha was found to possess $2 \mathrm{n}=42$ number of chromosomes along with a karyotype formula: $1 \mathrm{M}+31 \mathrm{~m}+8 \mathrm{sm}+2 \mathrm{st}$ using giemsa staining technique. The results demonstrated 'diffuse type of interphase nuclei, co-existence of continuous type and interstitial type of prophase chromosomes respectively. No heteromorphic sex chromosomes were determined cytologically. The presence of diverse types of chromosomes based on centromeric position, gradual decrease in total haploid chromosome complement, mean centromeric asymmetry, coefficient of variation of chromosome length and Stebbins's classification highlighted its asymmetry in karyotype with advance nature. Therefore, the elemental karyological data will offer information for the proper identification, cytotaxonomical classification, expanding productivity and preservation of genetic resources of T. ilisha.
\end{abstract}

Key words: Karyological data, Clupeidae family, Tenualosa ilisha, Padma and Meghna River, Bangladesh

\section{INTRODUCTION}

Tenualosa ilisha Family: Clupeidae) is a tropical anadromous fish mainly distributed from the Arabian Sea to the Persian Gulf and the Bay of Bengal and some estuaries, coastal regions and freshwater rivers (Blaber 2000, Arai and Amalina 2014) T. ilisha also familiar as Hilsha shad and recognized as the national fish of Bangladesh. They are the most dominating food fish in the countries within the Bay of Bengal (Rahman et al. 2018), contributing around

*Author for corresponding: <kazinahida.bot.jnu.ac.bd>, ${ }^{2}$ Department of Botany, Faculty of Life and Earth Sciences, Jagannath University, Dhaka-1100, Bangladesh

(C2021 Zoological Society of Bangladesh DOI: https://doi.org/10.3329/bjz.v49i2.56261 
$44 \%$ of the captured fish production, $10.50 \%$ of the total annual fish production, and over 1\% of the total gross domestic product of Bangladesh (DoF 2018) and to a limited extent in both India and Myanmar (Hossain et al. 2019, Rahman et al. 2018).T. ilisha contains a good amount of amino acids, lipids and minerals with flavorsome taste (Begum et al. 2016). T.ilisha renders beneficial effects for human health because of very high level of high density lipoprotein (HDL) and low level of low density lipoprotein (LDL) (De et al. 2019). It is commercially important due to its high content of omega fatty acid and the potentials for therapeutic applications (Dutta and Hazra 2017).

Recent study on $T$. ilisha of Bangladesh revealed a single panmictic population with an awful low genetic variation and facing breeding failure during migration and changing the pattern of spawning, which might cause experience of genetic bottleneck (Sarker et al. 2021). Elsewhere published data on T. ilisha focused on characterization, phenotypic traits, migratory habitats, salinity tolerance, breeding and evolution (Endler 1977, Hedrick 1986, Meyers and Bull 2002, Klemetsen 2010, Valiente et al. 2010, Stelkens et al. 2012). A considerable number of molecular analyses were performed globally (Das et al. 2018, Mahindra et al. 2019, Asaduzzaman et al. 2020, Sarker et al. 2021).

As cytogenetical analyses are inevitable as they provide important details based on numerical and structural characteristics of chromosome set as to karyotype (Akber et al. 2020, Saha et al. 2020). Only cytogenetical report highlighting the chromosomal aberration of T. ilisha was carried out in Iraq (Majeed 2016). None was focused on the karyotype study of $T$. ilisha from the confluence points of different rivers of Bangladesh. Therefore, the aim of this study was to perform karyotype study of T. ilisha from Padma and Meghna Rivers of Bangladesh for authentic identification characterization and conservation of fish genetic resources of $T$. ilisha.

\section{MATERIAL AND METHODS}

Sample collection: Freshly caught $T$. ilisha were collected with the help of local fishermen using fishing net from Chandpur, the confluence point of the Padma and Meghna Rivers stretched to the Bay of Bengal (Fig. 1). Chandpur was selected as a sample collection site due to the abundance and flavorful quality of $T$. ilisha.

Fish identification: Identification of $T$. ilisha was done following the taxonomic study of Rahman (2005). During sampling, the sexes could not be recognized phynotypically as the specimens were small in size. 


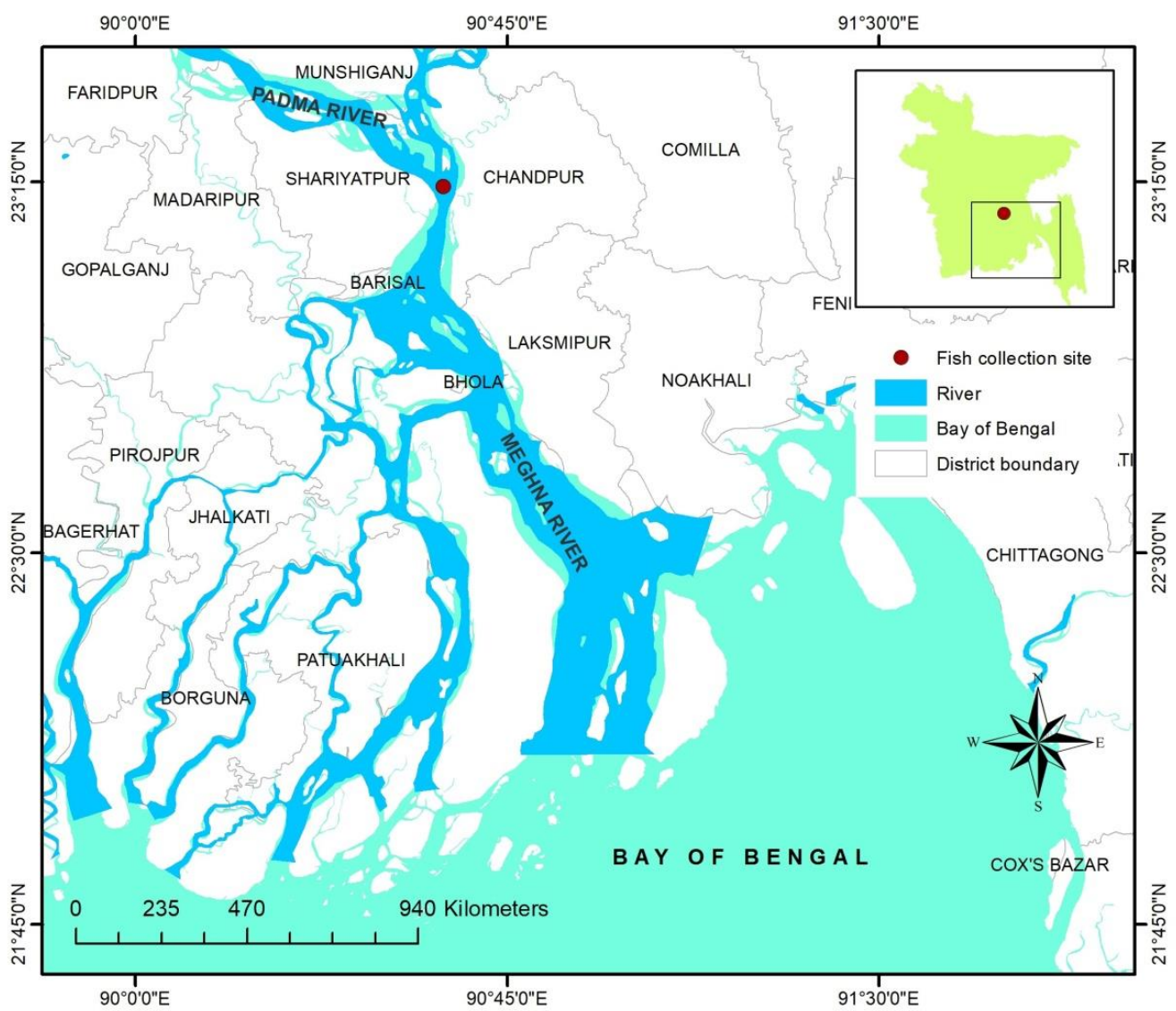

Fig. 1. Location of sampling sites in the confluence of Padma and Meghna River at Chandpur.

Fish sample preparation: Ten live fish were injected with $0.01 \%$ of Colcemid solution (Colcemid ${ }^{\mathrm{TM}}$, New York) at the dose of $0.02 \mathrm{mlg}^{-1}$ body weight of fish. After 30 minutes, the fish were sacrificed followed by collection of kidney cells and preparation of chromosomal suspension using elsewhere published data with slight modification (Bertollo et al. 2015). Firstly, the dissected kidney was kept within a hypotonic solution of $1 \%$ sodium citrate for 20 minutes, $1 \mathrm{gm}$ of grinded kidney was taken in a falcon tube containing $1 \mathrm{ml}$ of hypotonic solution and centrifuged (Centrifuge, China) at $2000 \mathrm{rpm}$ for 10 minutes. The precipitated cells were mixed with $2 \mathrm{ml}$ of Carnoy's fixative (1 acetic acid: 3 ethanol) and kept for 30 minutes. The fixed precipitated cells were again centrifuged at $2000 \mathrm{rpm}$ for 10 minutes. This process was repeated for three times with an interval of 30 minutes. At the end of centrifugation, $6 \mathrm{ml}$ of 
modified Carnoy's fixative ( 1 acetic acid: 1 ethanol) was mixed properly with the pellet and kept aside in a falcon tube for a while. The pellet was then shaken for few minutes and few drops of it were smeared on clean and dry slides. The slides were heated, air-dried, and dipped into 6\% Giemsa solution (Green Lab Chemicals, Bangladesh). Subsequently, the slides were rinsed gently with slow stream water to wash off excess stain and allowed to keep in open air for 30-40 minutes to dry. Again the slides were dipped into the xylene solution for 10-15 minutes. Finally, the prepared slides were observed under Optika electric microscope and mitotic stages (interphases, prophases and metaphases) were photographed with 40XS magnification using the Euromax camera (CMEX- 10, DC 10000C).

Data analysis: A total of 20 to 25 interphase nuclei and prophase chromosome were observed carefully to analyze the nature of heterochromatic in interphase nuclei and prophase chromosomes of $T$. ilisha and afterwards categorized on the basis of Tanaka's classification (1971). Twenty-five metaphase plates were observed and among them five well scattered somatic metaphases were considered to count chromosome number and preparation of karyotype and haploid idiogram. Karyotype was prepared considering the total length of homologous pair of diploid chromosome where a haploid idiogram was arranged in regard to the decreasing order of chromosome size. The chromosomes were categorized on the basis of centromeric position (Levan et al. 1964). The fundamental arm number (NF) was determined from the karyotype by attributing the value 2 to bi-armed chromosomes (metacentric and submetacentric) and value of 1 to uniarmed chromosomes (sub-telocentric and telocentric)

Statistical analysis: Mean centromeric asymmetry, MCA (Peruzzi and Eroğlu 2013), coefficient of variation of chromosome length, $C_{C L}$ (Paszko 2006), and Stebbins's classification (Stebbins 1971) were evaluated to characterize the karyological relationship in regard to karyotype asymmetry using a computer based software KaryoType_Win_2 (Altınordu et al. 2016).

\section{RESULTS AND DISCUSSION}

Giemsa-staining properties of interphase nuclei and prophase chromosomes unveiled that the interphase nuclei of $T$. ilisha were found to be uniformly stained demonstrating homogenous distribution of heterochromatins all over the nucleus. No prominent nucleolus was observed from the interphase nuclei of $T$. ilisha (Fig. 2a). On the other hand, co-existence of staining at interstitial area of prophase chromosomes along with continuously stained prophase chromosomes were observed in examined material of T. ilisha (Fig. 2b). 


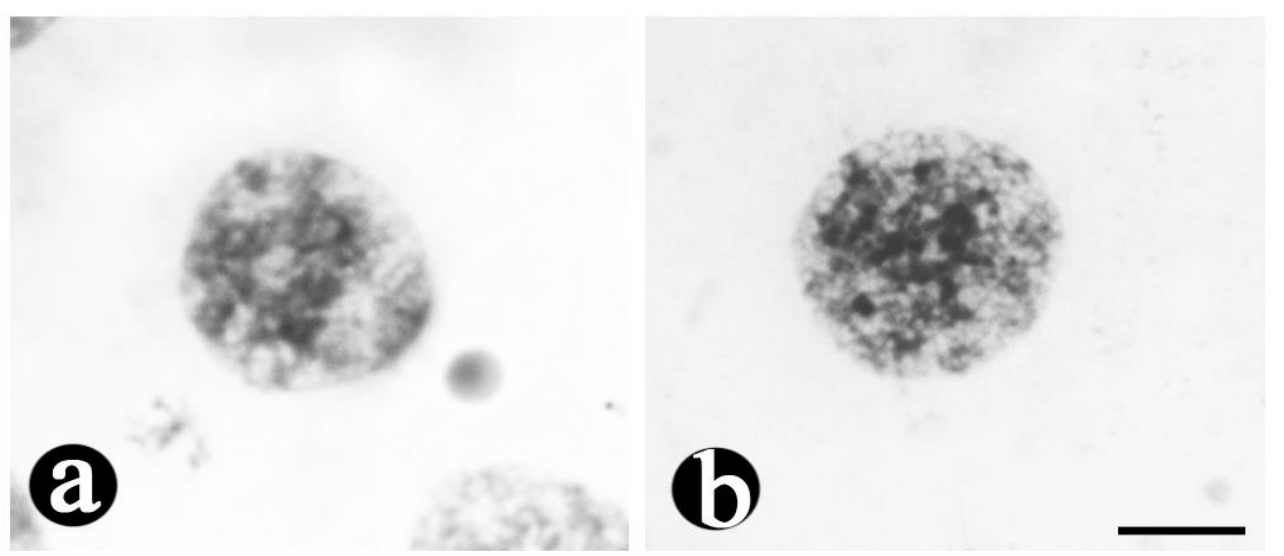

Fig. 2. Giemsa-stained mitotic interphase nuclei and prophase chromosomes of T. ilisha at 40XS magnification. (a) mitotic interphase nuclei and (b) mitotic prophase chromosomes. Scale bar $=5 \mu \mathrm{m}$.

Twenty-five mitotic metaphase plates from kidney tissues of T. ilisha were observed, of which 5 scattered mitotic metaphases were considered suitable for detailed karyological investigation. Mitotic metaphase stages demonstrated the somatic chromosome numbers $(2 \mathrm{n})$ and the karyotype was prepared from mitotic metaphases stages (Fig. 3a-b). The present study found diploid complement of $2 \mathrm{n}=42$ chromosomes with a perfectly metacentric chromosome (chromosome having long and short arm of equal length in pair no. 21), 31 metacentric chromosomes (in pair no. 1, 3-4, 6-7, 9, 12-21), 8 sub-metacentric chromosomes (in pair no. 2, 5, 8, 11) and 2 acrocentric chromosomes (in pair no. 10) and all of them exhibited with a karyotypic formula as: $1 \mathrm{M}+31 \mathrm{~m}+8 \mathrm{sm}$ +2 st. No morphologically different chromosomes related to sex were differentiated (Fig. 3a-b).
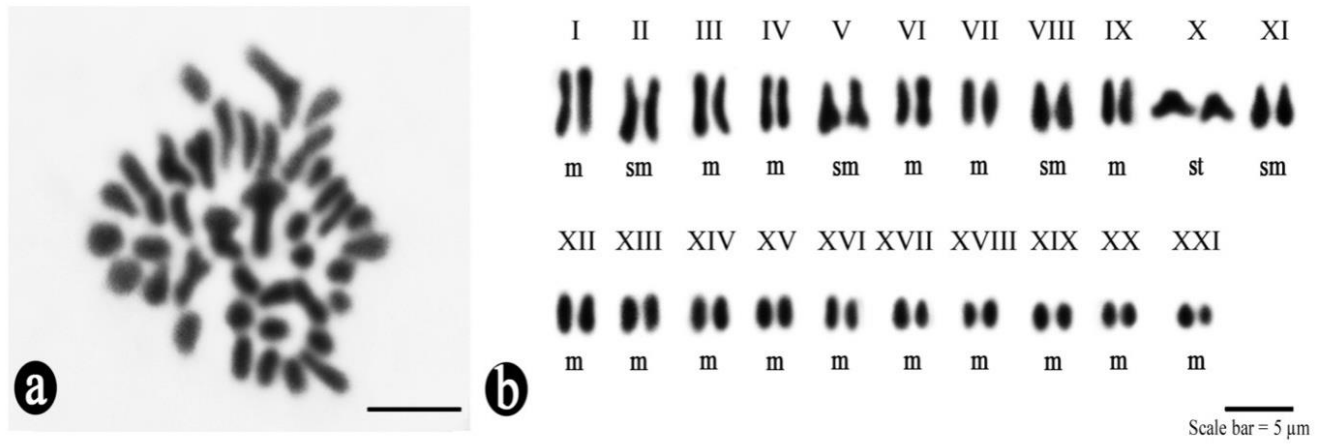

Fig. 3. Giemsa-stained mitotic metaphase chromosomes and karyotype of $T$. ilisha at 40XS magnification. (a) mitotic metaphase chromosomes and (b) karyotypes. Scale bars $=5 \mu \mathrm{m}$. 
The total length of diploid chromosome complement was $93.29 \pm 3.65 \mu \mathrm{m}$ with individual chromosomal length ranging from $1.18 \pm 0.35 \mu \mathrm{m}$ to $3.74 \pm 0.25$ $\mu \mathrm{m}$. Average chromosomal length was recorded $2.22 \mu \mathrm{m}$. The percentage of relative length for the smallest chromosome of the complement was 1.26 whereas the percentage of relative length for the largest chromosome was 4.01 (Table 1).

Table 1. Total length, arm ratio, relative length, centromeric index and centromeric type of mitotic metaphase chromosomes of $T$. ilisha

\begin{tabular}{|c|c|c|c|c|c|c|c|}
\hline $\begin{array}{c}\text { Chromosome } \\
\text { Pair }\end{array}$ & $\begin{array}{c}\text { Long } \\
\text { arm (1) } \\
\pm \mathrm{SD} \\
(\mu \mathrm{m})\end{array}$ & $\begin{array}{c}\text { Short } \\
\operatorname{arm}(\mathrm{s}) \\
\pm \mathrm{SD} \\
(\mu \mathrm{m})\end{array}$ & $\begin{array}{c}\text { Total } \\
\text { length } \\
(\mathrm{T}) \pm \mathrm{SD} \\
(\mu \mathrm{m})\end{array}$ & $\begin{array}{l}\text { Arm } \\
\text { ratio }\end{array}$ & $\begin{array}{c}\text { Relative } \\
\text { length } \\
\text { (RL) }\end{array}$ & $\begin{array}{c}\text { Centromeric } \\
\text { index (CI) }\end{array}$ & $\begin{array}{c}\text { Centromeric } \\
\text { type (CT) }\end{array}$ \\
\hline \multirow[t]{2}{*}{ I } & $\begin{array}{c}2.08 \pm \\
0.09\end{array}$ & $\begin{array}{l}1.66 \pm \\
0.16\end{array}$ & $\begin{array}{c}3.74 \pm \\
0.25\end{array}$ & 1.25 & 4.01 & 44.44 & $\mathrm{~m}$ \\
\hline & $\begin{array}{c}2.01 \pm \\
0.27\end{array}$ & $\begin{array}{l}1.71 \pm \\
0.15\end{array}$ & $\begin{array}{c}3.72 \pm \\
0.43\end{array}$ & 1.18 & 3.99 & 45.88 & $\mathrm{~m}$ \\
\hline \multirow[t]{2}{*}{ II } & $\begin{array}{c}2.20 \pm \\
0.18\end{array}$ & $\begin{array}{l}1.26 \pm \\
0.21\end{array}$ & $\begin{array}{c}3.45 \pm \\
0.32\end{array}$ & 1.75 & 3.70 & 36.42 & $\mathrm{sm}$ \\
\hline & $\begin{array}{c}2.16 \pm \\
0.23\end{array}$ & $\begin{array}{l}1.18 \pm \\
0.11\end{array}$ & $\begin{array}{c}3.35 \pm \\
0.47\end{array}$ & 1.83 & 3.59 & 35.29 & $\mathrm{sm}$ \\
\hline \multirow[t]{2}{*}{ III } & $\begin{array}{c}1.92 \pm \\
0.16\end{array}$ & $\begin{array}{l}1.40 \pm \\
0.17\end{array}$ & $\begin{array}{c}3.32 \pm \\
0.29\end{array}$ & 1.38 & 3.56 & 42.11 & $\mathrm{~m}$ \\
\hline & $\begin{array}{c}1.84 \pm \\
0.21\end{array}$ & $\begin{array}{l}1.27 \pm \\
0.19\end{array}$ & $\begin{array}{c}3.10 \pm \\
0.23\end{array}$ & 1.45 & 3.32 & 40.85 & $\mathrm{~m}$ \\
\hline \multirow[t]{2}{*}{ IV } & $\begin{array}{c}1.71 \pm \\
0.06\end{array}$ & $\begin{array}{l}1.26 \pm \\
0.11\end{array}$ & $\begin{array}{c}2.97 \pm \\
0.19\end{array}$ & 1.35 & 3.18 & 42.56 & $\mathrm{~m}$ \\
\hline & $\begin{array}{c}1.44 \pm \\
0.10\end{array}$ & $\begin{array}{l}1.31 \pm \\
0.08\end{array}$ & $\begin{array}{c}2.75 \pm \\
0.16\end{array}$ & 1.10 & 2.94 & 47.62 & $\mathrm{~m}$ \\
\hline \multirow[t]{2}{*}{ V } & $\begin{array}{c}1.83 \pm \\
0.13\end{array}$ & $\begin{array}{l}0.92 \pm \\
0.22\end{array}$ & $\begin{array}{c}2.75 \pm \\
0.63\end{array}$ & 2.00 & 2.94 & 33.39 & $\mathrm{sm}$ \\
\hline & $\begin{array}{c}1.78 \pm \\
0.15\end{array}$ & $\begin{array}{l}0.96 \pm \\
0.26\end{array}$ & $\begin{array}{c}2.74 \pm \\
0.28\end{array}$ & 1.85 & 2.93 & 35.06 & $\mathrm{sm}$ \\
\hline \multirow[t]{2}{*}{ VI } & $\begin{array}{c}1.34 \pm \\
0.09\end{array}$ & $\begin{array}{l}1.22 \pm \\
0.11\end{array}$ & $\begin{array}{c}2.56 \pm \\
0.14\end{array}$ & 1.09 & 2.74 & 47.78 & $\mathrm{~m}$ \\
\hline & $\begin{array}{c}1.31 \pm \\
0.12\end{array}$ & $\begin{array}{l}1.23 \pm \\
0.08\end{array}$ & $\begin{array}{c}2.55 \pm \\
0.24\end{array}$ & 1.06 & 2.73 & 48.45 & $\mathrm{~m}$ \\
\hline \multirow[t]{2}{*}{ VII } & $\begin{array}{c}1.36 \pm \\
0.16\end{array}$ & $\begin{array}{l}1.18 \pm \\
0.34\end{array}$ & $\begin{array}{c}2.55 \pm \\
0.55\end{array}$ & 1.16 & 2.73 & 46.39 & $\mathrm{~m}$ \\
\hline & $\begin{array}{c}1.31 \pm \\
0.11\end{array}$ & $\begin{array}{l}1.20 \pm \\
0.07\end{array}$ & $\begin{array}{c}2.51 \pm \\
0.20\end{array}$ & 1.09 & 2.69 & 47.74 & $\mathrm{~m}$ \\
\hline \multirow[t]{2}{*}{ VIII } & $\begin{array}{c}1.75 \pm \\
0.13\end{array}$ & $\begin{array}{l}0.74 \pm \\
0.18\end{array}$ & $\begin{array}{c}2.49 \pm \\
0.31\end{array}$ & 2.35 & 2.67 & 29.82 & $\mathrm{sm}$ \\
\hline & $\begin{array}{c}1.71 \pm \\
0.21\end{array}$ & $\begin{array}{l}0.79 \pm \\
0.10\end{array}$ & $\begin{array}{c}2.49 \pm \\
0.25\end{array}$ & 2.17 & 2.67 & 31.58 & $\mathrm{sm}$ \\
\hline \multirow[t]{2}{*}{ IX } & $\begin{array}{c}1.27 \pm \\
0.09\end{array}$ & $\begin{array}{l}1.20 \pm \\
0.17\end{array}$ & $\begin{array}{c}2.47 \pm \\
0.24\end{array}$ & 1.05 & 2.65 & 48.67 & $\mathrm{~m}$ \\
\hline & $\begin{array}{l}1.24 \pm \\
0.17\end{array}$ & $\begin{array}{l}1.18 \pm \\
0.18\end{array}$ & $\begin{array}{c}2.42 \pm \\
0.31\end{array}$ & 1.05 & 2.59 & 48.74 & $\mathrm{~m}$ \\
\hline \multirow[t]{2}{*}{$\mathrm{X}$} & $\begin{array}{c}1.75 \pm \\
0.11\end{array}$ & $\begin{array}{l}0.57 \pm \\
0.07\end{array}$ & $\begin{array}{c}2.32 \pm \\
0.14\end{array}$ & 3.08 & 2.49 & 24.53 & st \\
\hline & $\begin{array}{c}1.72 \pm \\
0.15\end{array}$ & $\begin{array}{l}0.53 \pm \\
0.35\end{array}$ & $\begin{array}{c}2.25 \pm \\
0.41\end{array}$ & 3.22 & 2.41 & 23.68 & st \\
\hline XI & $1.58 \pm$ & $0.67 \pm$ & $2.24 \pm$ & 2.36 & 2.40 & 29.73 & $\mathrm{sm}$ \\
\hline
\end{tabular}




\begin{tabular}{|c|c|c|c|c|c|c|c|}
\hline $\begin{array}{c}\text { Chromosome } \\
\text { Pair }\end{array}$ & $\begin{array}{c}\text { Long } \\
\text { arm (1) } \\
\pm \mathrm{SD} \\
(\mu \mathrm{m})\end{array}$ & $\begin{array}{c}\text { Short } \\
\operatorname{arm}(\mathrm{s}) \\
\pm \mathrm{SD} \\
(\mu \mathrm{m})\end{array}$ & $\begin{array}{c}\text { Total } \\
\text { length } \\
(\mathrm{T}) \pm \mathrm{SD} \\
(\mu \mathrm{m})\end{array}$ & $\begin{array}{l}\text { Arm } \\
\text { ratio }\end{array}$ & $\begin{array}{c}\text { Relative } \\
\text { length } \\
\text { (RL) }\end{array}$ & $\begin{array}{c}\text { Centromeric } \\
\text { index (CI) }\end{array}$ & $\begin{array}{c}\text { Centromeric } \\
\text { type (CT) }\end{array}$ \\
\hline \multirow{4}{*}{ XII } & 0.22 & 0.12 & 0.15 & & & & \\
\hline & $\begin{array}{c}1.48 \pm \\
0.09\end{array}$ & $\begin{array}{l}0.70 \pm \\
0.33\end{array}$ & $\begin{array}{l}2.18 \pm \\
0.47\end{array}$ & 2.11 & 2.34 & 32.16 & $\mathrm{sm}$ \\
\hline & $\begin{array}{c}1.18 \pm \\
0.17\end{array}$ & $\begin{array}{l}0.96 \pm \\
0.25\end{array}$ & $\begin{array}{l}2.14 \pm \\
0.19\end{array}$ & 1.23 & 2.29 & 44.90 & $\mathrm{~m}$ \\
\hline & $\begin{array}{c}1.22 \pm \\
0.13\end{array}$ & $\begin{array}{l}0.92 \pm \\
0.10\end{array}$ & $\begin{array}{l}2.14 \pm \\
0.27\end{array}$ & 1.33 & 2.29 & 42.86 & $\mathrm{~m}$ \\
\hline \multirow[t]{2}{*}{ XIII } & $\begin{array}{l}1.14 \pm \\
0.22\end{array}$ & $\begin{array}{l}0.92 \pm \\
0.24\end{array}$ & $\begin{array}{l}2.06 \pm \\
0.63\end{array}$ & 1.24 & 2.21 & 44.68 & $\mathrm{~m}$ \\
\hline & $\begin{array}{c}1.09 \pm \\
0.31\end{array}$ & $\begin{array}{l}0.83 \pm \\
0.11\end{array}$ & $\begin{array}{l}1.92 \pm \\
0.45\end{array}$ & 1.32 & 2.06 & 43.18 & $\mathrm{~m}$ \\
\hline \multirow[t]{2}{*}{ XIV } & $\begin{array}{l}1.01 \pm \\
0.17\end{array}$ & $\begin{array}{l}0.87 \pm \\
0.26\end{array}$ & $\begin{array}{l}1.88 \pm \\
0.18\end{array}$ & 1.15 & 2.02 & 46.51 & $\mathrm{~m}$ \\
\hline & $\begin{array}{l}1.01 \pm \\
0.14\end{array}$ & $\begin{array}{l}0.79 \pm \\
0.09\end{array}$ & $\begin{array}{l}1.79 \pm \\
0.56\end{array}$ & 1.28 & 1.92 & 43.90 & $\mathrm{~m}$ \\
\hline \multirow[t]{2}{*}{$\mathrm{XV}$} & $\begin{array}{c}0.92 \pm \\
0.27\end{array}$ & $\begin{array}{l}0.83 \pm \\
0.31\end{array}$ & $\begin{array}{l}1.75 \pm \\
0.21\end{array}$ & 1.11 & 1.88 & 47.50 & $\mathrm{~m}$ \\
\hline & $\begin{array}{c}0.87 \pm \\
0.24\end{array}$ & $\begin{array}{l}0.79 \pm \\
0.15\end{array}$ & $\begin{array}{l}1.66 \pm \\
0.13\end{array}$ & 1.11 & 1.78 & 47.37 & $\mathrm{~m}$ \\
\hline \multirow[t]{2}{*}{ XVI } & $\begin{array}{c}0.96 \pm \\
0.11\end{array}$ & $\begin{array}{l}0.66 \pm \\
0.23\end{array}$ & $\begin{array}{l}1.62 \pm \\
0.38\end{array}$ & 1.47 & 1.74 & 40.54 & $\mathrm{~m}$ \\
\hline & $\begin{array}{c}0.87 \pm \\
0.16\end{array}$ & $\begin{array}{l}0.70 \pm \\
0.09\end{array}$ & $\begin{array}{l}1.57 \pm \\
0.13\end{array}$ & 1.25 & 1.68 & 44.44 & $\mathrm{~m}$ \\
\hline \multirow[t]{2}{*}{ XVII } & $\begin{array}{c}0.91 \pm \\
0.07\end{array}$ & $\begin{array}{l}0.66 \pm \\
0.22\end{array}$ & $\begin{array}{l}1.57 \pm \\
0.27\end{array}$ & 1.39 & 1.68 & 41.78 & $\mathrm{~m}$ \\
\hline & $\begin{array}{l}0.82 \pm \\
0.23\end{array}$ & $\begin{array}{l}0.66 \pm \\
0.13\end{array}$ & $\begin{array}{l}1.48 \pm \\
0.36\end{array}$ & 1.25 & 1.59 & 44.44 & $\mathrm{~m}$ \\
\hline \multirow[t]{2}{*}{ XVIII } & $\begin{array}{c}0.79 \pm \\
0.21\end{array}$ & $\begin{array}{l}0.63 \pm \\
0.22\end{array}$ & $\begin{array}{l}1.42 \pm \\
0.25\end{array}$ & 1.24 & 1.52 & 44.62 & $\mathrm{~m}$ \\
\hline & $\begin{array}{c}0.77 \pm \\
0.16\end{array}$ & $\begin{array}{l}0.64 \pm \\
0.32\end{array}$ & $\begin{array}{l}1.41 \pm \\
0.49\end{array}$ & 1.19 & 1.51 & 45.65 & $\mathrm{~m}$ \\
\hline \multirow[t]{2}{*}{ XIX } & $\begin{array}{c}0.73 \pm \\
0.30\end{array}$ & $\begin{array}{l}0.67 \pm \\
0.27\end{array}$ & $\begin{array}{l}1.40 \pm \\
0.29\end{array}$ & 1.10 & 1.50 & 47.66 & $\mathrm{~m}$ \\
\hline & $\begin{array}{c}0.74 \pm \\
0.11\end{array}$ & $\begin{array}{l}0.66 \pm \\
0.24\end{array}$ & $\begin{array}{l}1.40 \pm \\
0.36\end{array}$ & 1.13 & 1.50 & 46.88 & $\mathrm{~m}$ \\
\hline \multirow[t]{2}{*}{$\mathrm{XX}$} & $\begin{array}{c}0.71 \pm \\
0.14\end{array}$ & $\begin{array}{l}0.66 \pm \\
0.11\end{array}$ & $\begin{array}{l}1.37 \pm \\
0.17\end{array}$ & 1.08 & 1.47 & 48.00 & $\mathrm{~m}$ \\
\hline & $\begin{array}{c}0.72 \pm \\
0.16\end{array}$ & $\begin{array}{l}0.61 \pm \\
0.09\end{array}$ & $\begin{array}{l}1.33 \pm \\
0.21\end{array}$ & 1.19 & 1.43 & 45.72 & $\mathrm{~m}$ \\
\hline XXI & $\begin{array}{c}0.63 \pm \\
0.07\end{array}$ & $\begin{array}{l}0.63 \pm \\
0.11\end{array}$ & $\begin{array}{l}1.27 \pm \\
0.15\end{array}$ & 1.00 & 1.36 & 50.00 & M \\
\hline & $\begin{array}{c}0.66 \pm \\
0.22\end{array}$ & $\begin{array}{l}0.52 \pm \\
0.13\end{array}$ & $\begin{array}{l}1.18 \pm \\
0.35\end{array}$ & 1.25 & 1.26 & 44.44 & $\mathrm{~m}$ \\
\hline GT & & & $\begin{array}{c}93.29 \pm \\
3.65\end{array}$ & & & & \\
\hline
\end{tabular}

$\mathrm{GT}=$ Grand total, $\mathrm{M}=$ perfectly metacentric, $\mathrm{m}=$ metacentric, $\mathrm{sm}=$ sub-metacentric, st $=$ subtelocentric chromosome

The number of fundamental chromosome arms was 82. The basic chromosome number was 21 . The total haploid chromosome length was $46.58 \pm$ $1.83 \mu \mathrm{m}$. The range of haploid chromosome complement was $1.26 \pm 0.25$ to 3.73 $\pm 0.34 \mu \mathrm{m}$ with arm ratio $(1.04-3.15)$ and relative length $(2.70-8.01 \%)$ (Fig. 4 
and Table 2). No secondary constriction was noticed from the examined specimens. The value of coefficient of variation of chromosome length $\left(\mathrm{CV}_{\mathrm{CL}}\right)$ and Mean centromeric asymmetry (MCA) was recorded 32.01 and 15.84 respectively and fall in 2B category of Stebbins's classification (Table 2).

Table 2. Different karyological features of the studied T. ilisha

\begin{tabular}{|c|c|c|c|c|c|c|c|c|c|c|c|}
\hline \multirow{2}{*}{$\begin{array}{l}\text { THCL } \\
\pm \text { SD } \\
(\mu \mathrm{m})\end{array}$} & \multicolumn{2}{|c|}{$\begin{array}{c}\text { HCL } \pm \text { SD } \\
(\mu \mathrm{m})\end{array}$} & \multicolumn{2}{|c|}{ Arm ratio } & \multicolumn{2}{|c|}{ RL (\%) } & \multirow[t]{2}{*}{ NF } & \multirow{2}{*}{$\mathrm{CV}_{\mathrm{CL}}$} & \multirow{2}{*}{ MCA } & \multirow{2}{*}{ SC } & \multirow{2}{*}{$\mathbf{K F}$} \\
\hline & Min & Max & Min & $\operatorname{Max}$ & Min & Max & & & & & \\
\hline $\begin{array}{c}46.58 \\
\pm \\
1.83 \\
\end{array}$ & $\begin{array}{l}1.26 \\
\pm \\
0.25\end{array}$ & $\begin{array}{l}3.73 \\
\pm \\
0.34\end{array}$ & 1.04 & 3.15 & 2.70 & 8.01 & 82 & 32.01 & 15.84 & $2 \mathrm{~B}$ & $\begin{array}{l}1 \mathrm{M}+31 \mathrm{~m} \\
+8 \mathrm{sm}+ \\
2 \mathrm{st}\end{array}$ \\
\hline
\end{tabular}

THCL $=$ Total haploid chromosomal length,$H C L=$ Haploid chromosomal length, RL $=$ Relative length, $\mathrm{NF}=$ Fundamental arm number, $\mathrm{CV}_{\mathrm{CL}}=$ Coefficient of variation of chromosome length, $\mathrm{MCA}=$ Mean centromeric asymmetry, $\mathrm{SC}=$ Stebbins's classification, $\mathrm{KF}=$ Karyotype formula.

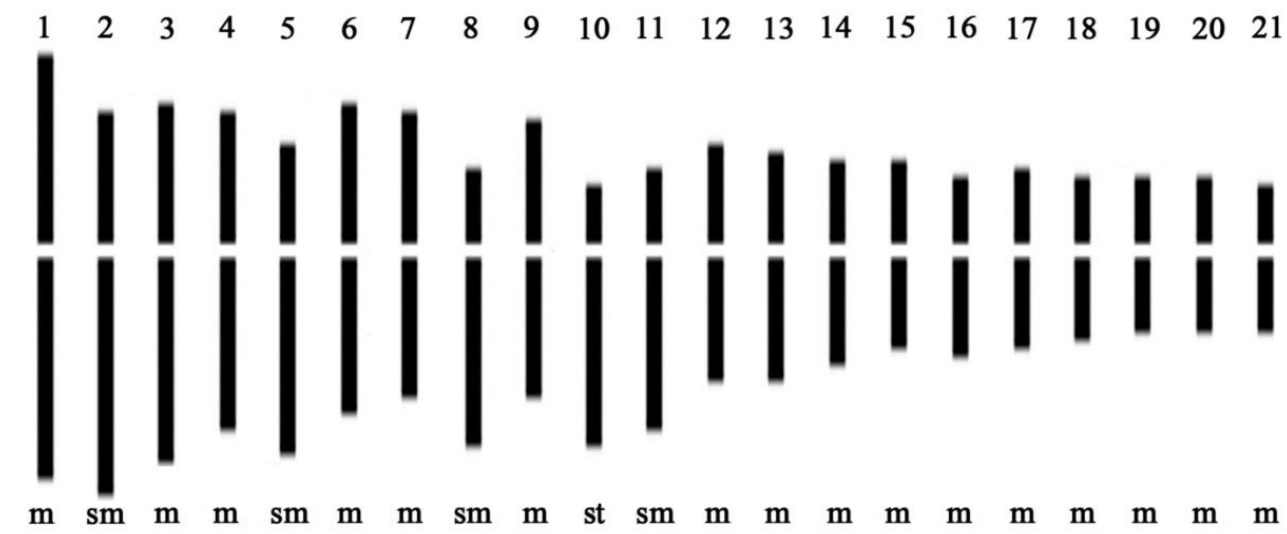

Scale bar $=5 \mu \mathrm{m}$

Fig. 4. Haploid idiogram of T. ilisha. Scale bar $=5 \mu \mathrm{m}$.

The staining properties of interphase nuclei and prophase chromosomes sometimes provide additional karyomorphological features that help to characterize different specimens (Tanaka 1971). In the present study, the nuclei were found uniformly stained and regarded as diffuse type of interphase nuclei (Tanaka 1971). Similarly, the prophase chromosomes were observed homogeneously stained throughout the nuclei but the interstitial areas were characterized by darkly stained heterochromatic blocks and referred to as continuous type and interstitial type of prophase chromosomes respectively 
(Tanaka 1971). Generally, the continuously stained heterochromatins as observed in the interphase nuclei were usually homogeneously distributed in the prophase chromosomes rather occupied at different locations. The present findings did not support the usual regulation in regard to the distribution of heterochromatin in prophase chromosomes. This study assumed the presence of constitutive heterochromatin blocks along with facultative heterochromatins might be the reasons for the chromosomal aberrations in the prophase chromosomes of $T$. ilisha.

Cytogenetical investigations revealed that $T$. ilisha shared a chromosome complement of $2 \mathrm{n}=42$ number of diploid chromosomes. T. ilisha from diverse topographical regions retained diploid chromosome count $(2 n)$ resulting changes in chromosome morphology rather alteration in chromosome number. The finding of this study was consistent with the earlier reportMajeed, 2016.

Moreover, in this study, we found 16 pair of metacentric chromosomes, 4 pair of sub-metacentric and 1 pair of sub-telocentic chromosomes (Table 2) which was different from the previous study (Majeed 2016). Considering the Robertsonian translocation (centric fusion of two acrocentric chromosomes and pericentric inversions) might cause differentiation in karyotype formula and also serves as stimulants all through the evolutionary pathway of karyotype diversification (Morgan-Richards 2000)

Kaewsri et al. (2014) stated the occurrence of pericentric inversion as responsible for higher amount of fundamental arm number. The presence of high fundamental arm number in this study reflects the pericentric inversion occurred intensely within the karyotype evolution of T. ilisha.

In this investigation, no heteromorphic pairs of chromosomes were distinguished as sex chromosomes which were opposite to the previous study (Majeed, 2016), underscore the reasons of small sized specimens caught. In other way, the methodology followed in the experiment might not be compatible for the determination of sex chromosomes could be regarded as the limitation of the study.

A graphical representation of haploid chromosome complement on a bidimensional scattered plot was presented in Fig. 5. From the scattered plot, it was observed that all the metacentric chromosomes (chromosome no. 1, 3-4, 67,9 and 12-21) were located on the lower portion $(1.00-1.50 \mu \mathrm{m})$ of the plot; whereas the only sub-telocentric chromosome (chromosome no. 10) was found on the upper most position of the plot $(3.0 \mu \mathrm{m})$ and the sub-metacentric chromosomes (chromosome no. 2, 5, 8 and 11) were observed on the middle portion of the plot (1.75 to $2.25 \mu \mathrm{m}$ ). 


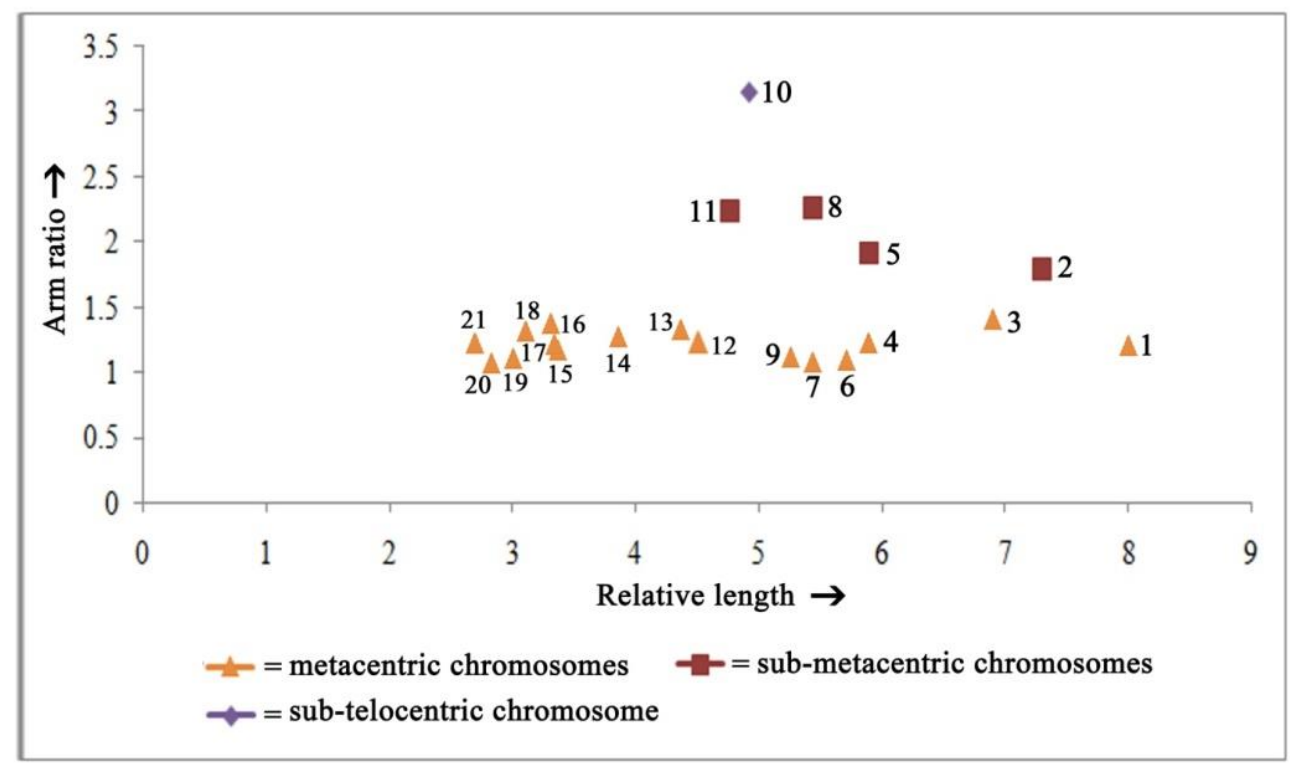

Fig. 5. Scattered diagram of haploid chromosomes of $T$. ilisha showing the position of different types of chromosomes (metacentric, sub-metacentric and sub-telocentric chromosomes).

In this study, $T$. ilisha showed karyotype formula of $1 \mathrm{M}+31 \mathrm{~m}+8 \mathrm{sm}+2 \mathrm{st}$ depending on the position of centromere and ratio of arms (Table 2). The karyotype formula exhibited with the combination of perfectly metacentric, metacentric, sub-metacentric and sub-telocentric chromosomes clearly explain the attribute of asymmetry in karyotype of T. ilisha from Padma and Meghna River confluence points of Bangladesh.

The average total length of haploid chromosome complement (THCL) was determined as $46.58 \pm 1.83 \mu \mathrm{m}$ ranging from $1.26 \pm 0.25 \mu \mathrm{m}$ to $3.73 \pm 0.34 \mu \mathrm{m}$ (Table 2). The haploid chromosome complement of our considered species demonstrated a clear continuous decrease because the difference in THCL between the shortest $(1.26 \mu \mathrm{m})$ and the longest chromosomes $(3.73 \mu \mathrm{m})$ by 2.5 folds $(2.47 \mu \mathrm{m})$ (Fig. 4). Presence of progressive diminished in length of chromosome complements was indicative asymmetry in karyotype.

In the prevailing study, an in depth observation was conveyed to characterise $T$. ilisha karyomorphologically and manifest evolution of karyotype. Presence of sub-metacentric and sub-telocentric chromosomes was conjointly reflected by MCA value (15.84) of Peruzzi and Eroglu (Peruzzi and Eroglu 2013) which confers to intrachromosomal asymmetry of chromosomes. For this reason the karyotype was categorized as Stebbins' category 2 of asymmetry (Table 2). The $32.01 \mathrm{CV}_{\mathrm{CL}}$ value by Paszko (2006), presenting 
interchromosomal asymmetry of chromosome complement was interpreted by more than two times length gap between the smallest $(1.26 \mu \mathrm{m})$ and the longest chromosome $(3.73 \mu \mathrm{m})$. Thus the karyotype comes under the asymmetry group B of Stebbins (Stebbins 1971). In accordance to Stebbins (1971), asymmetry of karyotype holds characters of advancement and can indeed be considered as the key catalyst behind speciation. Therefore it could be taken into consideration that $T$. ilisha might have possessed advance characters.

\section{CONCLUSION}

The karyological data on T. ilisha revealed $2 \mathrm{n}=42$ number of chromosomes with detailed karyomorphology could play a contributory role in proper identification and classification of $T$. ilisha and may provide basic cytological information for the enhancement of sustainable productivity of T. ilisha through molecular technologies of breeding.

Acknowledgement: The financial support from the Ministry of Science and Technology, Government of the People's Republic of Bangladesh is gratefully acknowledged by the first author. The authors are also thankful to the Department of Botany (Molecular Cytogenetics), Jagannath University for providing the laboratory facilities and their support throughout the entire research work.

\section{LITERATURE CITED}

AKBAR, F., PAUL, M. and BEGUM, K.N. 2020. Karyological and genetic diversity study using molecular marker among three species of oilseed Brassica L. Ind. J. Pure \& App. Biosci. 8 (4): 267-281.

ALTINORDU, F., PERUZZI, L., YU, Y. and HE, X. 2016. A tool for the analysis of chromosomes: KaryoType. Taxon, 65 (3): 586-592.

ARAI, T. and AMALINA, R. 2014. New record of a tropical shad Tenualosa ilisha (Teleostei: Clupeidae) in Malaysian waters. Mar. Biodivers. Rec.7: 1-4.

ASADUZZAMAN, M., IGARASHI, Y., WAHAB, M.A., NAHIDUZZAMAN, M., RAHMAN, M.J., PHILLIPS, M.J., HUANG, S., ASAKAWA, S., RAHMAN, MD.M. and WONG, L.L. 2020. Population genomics of an anadromous Hilsa Shad, Tenualosa ilisha species across its diverse migratory habitats: discrimination by fine-scale local adaptation. Genes 11 (1): 1-23.

BEGUM, M., BHOWMIK, S., JULIANA, F.M., and HOSSAIN, Md.S. 2016. Nutritional Profile of Hilsa Fish Tenualosa ilisha (Hamilton, 1822) in six selected regions of Bangladesh. J. Nutr. Food Sci. 6 (2): $567-570$.

BERTOLLO, L.A.C., CIOFFI, M. and MOREIRA-FILHO, O. 2015. Direct chromosome preparation from freshwater teleost fishes. Fish Cytogenetic Techniques (Chondrichthyans and Teleosts); 
Ozouf-Costaz, C., Pisano, E., Foresti, F., Almeida Toledo, L.F., Eds, CRC Press, Florida, 21-26 pp.

BLABER, S.J.M. 2000. Tropical estuarine fishes: Ecology, exploitation and conservation. WileyBlackwell, Oxford, England. 384 pp.

DAS, A., LANAKIEV, P., BATEN, A., NEHLEEN, R., EHSAN, T., AHMED, O., ISLAM, Md.R., NASER, M.N., MARMA, M.S. and KHAN, H. 2018. Genome of Tenualosa ilisha from the River Padma, Bangladesh. BMC Research Notes 11 (1): 1-3.

DE, D., MUKHERJEE, S., SHYNE ANAND, P.S., KUMAR, P., SURESH, V.R. and VIJAYAN, K.K. 2019. Nutritional profiling of hilsa (Tenualosa ilisha) of different size groups and sensory evaluation of their adults from different riverine systems. Scientific Report, 9: 1-11.

DoF (Department of Fisheries) 2018. Yearbook of Fisheries Statistics of Bangladesh, 2017-18. Fisheries Resources Survey System (FRSS), Department of Fisheries Bangladesh: Ministry of Fisheries and Livestock Government of the People's Republic of Bangladesh. Vol. 35, 129 pp.

DUTTA, S. and HAZRA, S. 2017. From biology to management: A critical review of Hilsa Shad (Tenualosa ilisha). Ind. J. Geo-Mar. Sci. 46 (8): 975-1033.

ENDLER, J.A. 1977. Geographic variation, speciation and clines. Princeton University Press.Princeton, New Jersey, USA. Vol. 10, 262 pp.

HEDRICK, P.W. 1986. Genetic polymorphism in heterogeneous environments: A decade later. Annu. Rev. Ecol. Evol. Syst. 17 (1): 535-566.

HOSSAIN, M.S., SHARIFUZZAMAN, S.M., ROUF, M.A., POMEROY, R.S., HOSSAIN, Md.D., CHOWDHURY, S.R. and AFTABUDDIN, S. 2019. Tropical hilsa shad (Tenualosa ilisha): Biology, fishery and management. Fish \& Fisheries 20 (1): 44-65.

KAEWSRI, S., TANOMTONG, A., GETLEKHA, N., SAENJUNDAENG, P., SUKSUWAN, R. and SUPIWONG, W. 2014. Standardized karyotype and idiogram of Quoy's Parrotfish, Scarusquoyi (Perciformes: Scaridae) by conventional staining and Ag-NOR banding techniques. Cytologia 79 (4): 429-435.

KLEMETSEN, A. 2010. The charr problem revisited: Exceptional phenotypic plasticity promotes ecological speciation in postglacial lakes. Freshwater Reviews, 3 (1): 49-75.

LEVAN, A., FREDGA, K. and SANGBERG, A.A. 1964. Nomenclature for centromeric position on chromosomes. Hereditas 52 (2): 201-220.

MAJEED, M.H. 2016. Chromosomal study of Tenualosa ilisha fish from Shatt-Al-Arab River. J. Env. Biosci. 30 (1): 59-60.

MEYERS, L.A. and BULL, J.J. 2002. Fighting change with change: adaptive variation in an uncertain world. Trends in Ecology \& Evolution, 17 (12): 551-557. MOHINDRA, V., DANGI, T., TRIPATHI, R.K., KUMAR, R., SINGH, R.K., JENA, J.K. and MOHAPATRA, T. 2019. Draft genome assembly of Tenualosa ilisha, Hilsa shad, provides resource for osmoregulation studies. Scientific reports, 9 (1): 1-14. 
MORGAN-RICHARDS, M. 2000. Robertsonian translocation and B chromosome chromosomes in the Willington tree weta, Hemideina crassidens (Orthoptera: Anostostomatidae). Heretidas 132 (1): 49-54

PASZKO, B. 2006. A critical review and a new proposal of karyotype asymmetry indices. Plant Syst. Evol. 258: 39-48.

PERUZZI, L. and EROĞLU, H.E. 2013. Karyotype asymmetry: again, how to measure and what to measure?. Comparative cytogenetics, 7(1): 1-9.

RAHMAN, A.K.A. 2005. Freshwater Fishes of Bangladesh, 2nd ed., Zool. Soc. Bangladesh, Dhaka, Bangladesh, 394 pp.

RAHMAN, M.J., WAHAB, M.A., AMIN, S.M.N., NAHIDUZZAMAN, M. and ROMANO, N. 2018. Catch trend and stock assessment of Hilsa Tenualosa ilisha using digital image measured lengthfrequency data. Mar. Coast. Fish. 10 (4): 386-401.

SAHA, S., PINKY, M.S., AKTER, S. and BEGUM, K.N. 2020. Karyotype diversity among twelve varieties of Brassica L. (Brassicaceae) from Bangladesh. Trop. Plant Res.7 (2): 476-483.

SARKER, A., JIANG, J., NAHER, H., HUANG, J., SARKER, K.K., YIN, G., BAKI, Md.A. and LI, C. 2021. Cross-species gene enhancement revealed a single population of Hilsha shad (Tenualosa ilisha) with low genetic variation in Bangladesh water. Scientific Report, 11 (1): 1 14.

STEBBInS, G.L. 1971. Chromosomal Evolution in Higher Plants. Edward Arnold, London, New York, $216 \mathrm{pp}$.

STELKENS, R.B., JAFFUEL, G., ESCHER, M. and WEDEKIND, C. 2012. Genetic and phenotypic population divergence on a microgeographic scale in brown trout. Molecular Ecology, 21 (12): 2896-2915.

TANAKA, R. 1971. Types of resting nuclei in Orchidaceae. Shokubutsugaku Zasshi, 84 (993): 118122.VALIENTE, A.G., JUANES, F., NUÑEZ, P. and GARCIA-VAZQUEZ, E. 2010. Brown trout (Salmo trutta) invasiveness: plasticity in life-history is more important than genetic variability. Biological Invasions, 12 (3): 451-462.

(Manuscript received on 25 June, 2021 revised on 25 August, 2021) 